explained by the fact that it is shielded against photolysis by methane which strongly absorbs solar photons above the $0.1 \mathrm{mbar}$ atmospheric level. HCN could be lost by downward transport followed by condensation around the $130 \mathrm{~K}$ temperature level, but the vertical mixing time is in the range 3 to 30 years in the stratosphere of Jupiter. Therefore, barring any major dilution due to planetwide transport, we expect to find HCN in Jupiter's stratosphere at about the same mole fraction for months or years. Observations of this species with the JCMT will be pursued in 1995.

\title{
THE COLLISION OF COMET P/SHOEMAKER-LEVY 9 WITH JUPITER: DETECTION AND MONITORING OF CO, CS AND OCS
}

E. Lellouch (Obs. Paris), G. Paubert, R. Moreno (IRAM), M.C. Festou (Obs. Midi-Pyrénées), B. Bézard, D. Bockelée-Morvan, P. Colom, J. Crovisier, T. Encrenaz, D. Gautier, A. Marten (Obs. Paris), D. Despois (Obs. Bordeaux), D.F. Strobel (Johns Hopkins U., Baltimore), A. Sievers (IRAM)

We report here on millimeter wave observations performed at the Institut de Radio Astronomie Millimétrique (IRAM) 30-m telescope at Pico Veleta, Spain, on July 17-28, 1994, to search for molecular and ion species that could plausibly be injected or generated in Jupiter's atmosphere by the impacts. Spectral resolutions ranged from 0.04 to $1 \mathrm{MHz}$. The telescope beam (12" full width at half-maximum (FWHM) at $230 \mathrm{GHz}$ ) did not resolve the impact sites and usually encompassed several sites after the first observing days.

Three molecules were detected: $\mathrm{CO}$ at $230.538 \mathrm{GHz}$ on impact sites $\mathrm{C}$, $\mathrm{E}, \mathrm{G}, \mathrm{H}, \mathrm{K}, \mathrm{L}, \mathrm{G}+\mathrm{Q}$ and $\mathrm{G}+\mathrm{Q}+\mathrm{R}+\mathrm{S} ; \mathrm{CS}$ at $244.935 \mathrm{GHz}$ on impact sites $\mathrm{K}$, $\mathrm{L}, \mathrm{G}+\mathrm{Q}+\mathrm{R}+\mathrm{S}$ and $\mathrm{K}+\mathrm{W}$ on July 21 and later; and $\mathrm{OCS}$ at $218.903 \mathrm{GHz}$ on July 22 at impact site $\mathrm{K}+\mathrm{W}$. CS and OCS were actually detected as soon as we attempted to observe them. $\mathrm{CO}$ was also detected at $115.271 \mathrm{GHz}$ on the "G+Q+R+S complex" on July 23.

Monitoring of the CO and CS lines was performed for a few impact sites. Until July 25, all lines appeared as narrow emission features (about $2.5-3 \mathrm{MHz}$ (or $3-4 \mathrm{~km} / \mathrm{s}$ ) FWHM). Linewidths remained fairly constant. Line intensities generally declined over a few days. In some cases however maximum intensities were not reached immediately after impact but rather 1-2 days later. On July 28, CO and CS were detected in absorption (with possibly, in the case of $\mathrm{CO}$, a narrower emission core). We interpret the initial intensity increase as caused by the expansion of the impact sites, while the subsequent decrease and the 
switch over to the absorption regime are primarily due to the cooling of the gas. Line positions for the $\mathrm{G}+\mathrm{Q}+\mathrm{R}+\mathrm{S}$ complex show that a large fraction of the emission comes from the $R$ site, although some frequency mismatches occur, presumably indicative of a multi-site emission.

The observed transitions correspond to thermal absorption/emission of Jupiter's atmosphere modified by the impacts. The linewidths must reflect a combination of pressure (Lorentz) broadening (thermal Doppler broadening would indicate an implausible temperature of $\sim 5000 \mathrm{~K}$ hours or days after the impacts) and velocity smearing. Pressure broadening would correspond to a reasonable formation level of $\sim 0.5 \mathrm{mbar}$. Velocity smearing inside the impact sites is probably not the dominant effect.

Observations were modelled by means of a radiative transfer code in which the minor species were assumed to be uniformly mixed above a pressure level essentially determined by the linewidths. On site $\mathrm{G}, \sim 10$ hours after impact, we find that the stratosphere near $0.5 \mathrm{mbar}$ had an excess temperature of at least $\Delta T=25-30 \mathrm{~K}$ over its normal $170 \mathrm{~K}$ temperature. The associated $\mathrm{CO}$ mixing ratio is $q_{C O}=4 \times 10^{-5}$ at $p \leq 0.3 \mathrm{mbar}$, corresponding to a CO mass of $\sim 1 \times 10^{14} \mathrm{~g}$. On site $\mathrm{G}+\mathrm{Q}+\mathrm{R}+\mathrm{S}$, the evolution of the CO lines on July 21,23 , 25 and 28 implies temperature variations relative to the "quiescent" profile of $\Delta T=+28 K,+10 K,-3 K$ and $-15 K$ respectively. Similarly analyzing the CS and OCS lines, we find that the largest impactors $(G, K)$ also generated $\sim 3 \times 10^{12} \mathrm{~g}$ of OCS and $3 \times 10^{11} \mathrm{~g}$ of CS. Interestingly, the O/S ratio inferred from these numbers is 70 (in volume), essentially solar. We also note that our CS abundance is about 500 times larger that than inferred from an an HST/FOS spectrum of plume $S, \sim 30$ minutes after impact.

The $\mathrm{CO}$ mixing ratio we derive, 50000 times larger than the normal Jupiter CO abundance, indicates that the oxygen seen in CO must be of cometary origin. However, the high temperatures generated by the impacts are expected to dissociate most of the cometary (including $\mathrm{CO}$ ) and jovian material in the fireball. Shock chemistry seems to provide the most plausible way of (re)forming molecular species. Zahnle and McLow estimate that, for a $1 \mathrm{~km}$ diameter cometary fragment with solar composition, about $10^{14} \mathrm{~g}$ of $\mathrm{CO}$ (along with similar amounts of $\mathrm{H}_{2} \mathrm{O}$ and much smaller masses of $\mathrm{CO}_{2}$ ) and a few times $10^{12} \mathrm{~g}$ of OCS are formed by shock chemistry, in essential agreement with our measurements. Their model predicts the formation of only small amounts of CS but significant $\mathrm{CS}_{2}$ abundances. The CS we are seeing may therefore be photochemically produced from $\mathrm{CS}_{2}$.

Our measurements imply that Jupiter's mid-stratospheric thermal profile remained modified a long time after the impacts. This effect may be primarily due to the injection, into the stratosphere, of considerable amounts of minor 
compounds, some of which efficiently radiate in the thermal ranges $\left(\mathrm{H}_{2} \mathrm{O}\right.$, $\mathrm{NH}_{3}, \mathrm{HCN}$...).

\section{OBSERVATIONS OF GALILEAN SATELLITES DURING THE COLLISIONS OF SHOEMAKER-LEVY 9 FRAGMENTS WITH JUPITER}

V. Ivanova, A. Antov, B. Komitov, V. Shkodrov, R. Antova, D. Kolev, Z. Donchev (Department of Astronomy, Bulgarian Academy of Sciences)

Observations of reflected light from Galilean satellites were carried out at Rozhen and Belogradchik observatories in Bulgaria during the collisions of $A, H$ and $Q$ fragments of Shoemaker-Levy 9 with Jupiter. The weather was unstable and often affected by clouds. $\mathrm{V}$ and $\mathrm{U}$ photoelectric observations were taken by $60 \mathrm{~cm}$ telescopes of Rozhen and Belogradchik observatories with an integration time of $1 \mathrm{sec}$. In parallel, at Belogradchik observatory, CCD photometry in $\mathrm{H}$-alpha was made with a $0.35 \mathrm{~m}$ Celestron telescope. With the Coude spectrograph at the $2 \mathrm{~m}$ telescope spectra of Io were taken in a range of $60 \mathrm{~A}$ around $\mathrm{H}$-alpha with a spectral resolution of $0.1 \mathrm{~A} /$ pixel $(1$ pixel $=23$ microns). The exposure time of the spectra was $10 \mathrm{~min}$. The reduction was made but no significant difference could be detected in different spectra.

Here we present the analysis of the photoelectric light curve of Callisto taken with a V-Johnson filter on July 18 (UT: 19:07-20:45) at Belogradchik during the $\mathrm{H}$ impact. Peculiar variations in the bright- ness can be seen with an accuracy of $+-0.03-+-0.05 \mathrm{mag}$. The amplitude of these variations is about $0.4 \mathrm{mag}$. Several rapid variations in the brightness appear at the beginning of the light curve, but later the increase of the brightness follows a steady rise. While the first peak could be considered within the noise level of the mean relative brightness, the next two peaks $A$ and $B$ at 19:31 and 19:45, respectively, are obviously real. Maximum of the brightness is at 20:10 and then it begins to decrease lightly. These changes in the light curve of Callisto during $\mathrm{H}$ impact were confirmed by parallel CCD-observations in $\mathrm{H}$-alpha, where the above mentioned peaks $A$ and $B$ are sharply marked and precede those in $\mathrm{V}$ by about 5 minutes. Moreover, the ratio of the drop after the second minimum to the drop after the first one is equal for both kinds of observations. Probably more than one piece has hit Jupiter during this impact.

The analysis of the observations continues. 